\title{
INVESTIGAÇÃO PANORÂMICA SOBRE A ADOÇÃO DE ESTRUTURAS FACETADAS NO VIÉS DA ARQUITETURA DA INFORMAÇÃO
}

\section{PANORAMIC INVESTIGATION ON THE ADOPTION OF FACETED STRUCTURES WITHIN THE VIEW OF INFORMATION ARCHITECTURE}

Márcio Bezerra da Silva ${ }^{a}$

Zeny Duarte de Mirandab

\begin{abstract}
RESUMO
Introdução: Considerando os sistemas de organização da arquitetura da informação, com ênfasenas estruturas de organização, discute-se a adoção de facetas em ambientes digitais, ponderandomodelos de facetação, suportes digitais onde os modelos foram adotados, modelos por escopo de atuação, evolução dos ambientes digitais ao longo dos anos e facetas à luz das categorias fundamentais PersonalidadeMatéria-Energia-Espaço-Tempo, oriundas da teoria da classificação facetada do indiano Ranganathan. Objetivo: Apresentar um panorama de uso de facetas na estruturação de ambientes digitais. Metodologia:Estudo exploratório e bibliográfico, com coleta de dados qualitativa de ambientes digitais que utilizam modelos de facetação e suas características, realizada em 2017 e continuada até 2020. Resultados: Os websites de e-commerce são os espaços onde os modelos facetados são mais adotados, tendo o wine.com como uma inspiração, inclusive com alguns atualizando suas facetações em novas perspectivas com o tempo; o escopo de aplicação de facetações concentra-se no mundo dos negócios, iniciando em 2006 e continuando ao longo do tempo; e personalidade foi a mais presente entre os itens das categorias fundamentais. Conclusões: A adoção de facetas permite que um mesmo objeto tenha interpretações diferentes em um menu navegacional, formaliza a multidimensionalidade idealizada por Ranganathan a partir de mnemônicas personalizadas ao escopo do ambiente digital, orienta na construção de taxonomias múltiplas e rasas e representa uma forma de classificação que acentua-se ao longo dos anos, fortalecendo a inferência de que os websites assumiram a facetação como um caminho para organizar a informação segundo estruturas de navegação e busca integradas.
\end{abstract}

Descritores: Facetas. Categorias Fundamentais. Teoria da Classificação Facetada.

\footnotetext{
a Doutor em Ciência da Informação pela Universidade Federal da Bahia (2018). Professor da Faculdade de Ciência da Informação $(\mathrm{FCl})$ da Universidade de Brasília (UnB). E-mail: marciobdsilva@unb.br

b Doutora em Letras pela Universidade Federal da Bahia (UFBA). Docente do Instituto de Ciência da Informação (ICl) da Universidade Federal da Bahia (UFBA). E-mail: zenydu@gmail.com
} 
Arquitetura da Informação. Estruturas de Organização.

\section{INTRODUÇÃO}

Quando se pensa na organização da informação (OI), enquanto "[...] um processo de arranjo de acervos tradicionais ou eletrônicos realizados por meio da descrição de assunto de seus objetos informacionais" (LIMA; ALVARES, 2012, p. 35), cria-se um direcionamento natural às ambições da organização do conhecimento (OC), afinal, apresenta-se como o subsídio "[...] mais importante na fundamentação teórica da organização da informação [...] baseada em unidades do conhecimento [...]" (DAHLBERG, 1993, p. 211), aqui invocados como conceitos, ou seja, "[...] enunciados verdadeiros sobre determinado objeto, fixada por um símbolo linguístico" (DAHLBERG, 1978, p. 102).

Na tentativa de realizar a OI, o campo da OC intenciona "[...] permitir que os usuários encontrem informações relevantes" (HJØRLAND, 2008, p. 91) e, assim, permiti-los editar e/ou ampliar a compreensão de mundo. Para tanto, elenca-se a ação chamada de classificação, compreendida por Dahlberg (2006) como sendo uma espécie de proximidade, similaridade ou relação de diferentes objetos que podem ser agrupados. Essa forma de organizar é a essência de se formar classes. Dahlberg (1979) já destacava que a classificação se mostrava como uma teoria que deixou de ser arte e progrediu ao status de ciência.

Independente do sistema de organização do conhecimento (SOC) adotado, a classificação objetiva "[...] mapear o universo multidimensional dos assuntos [...]" (RANGANATHAN, 1967, p. 395), operando, em suma, da seguinte forma: atuar como um meio na confecção de relações entre os campos do conhecimento humano; relacionar conceitos/termos/ícones em disciplinas, línguas e culturas; fornecer a base conceitual para o design e implementação de pesquisas; prover suporte na recuperação de informações, com base no conhecimento do usuário no momento da pesquisa, a partir de estruturas em árvores, análise facetada, navegação hierárquica ou mapa conceitual (MC), os identificando conceitos, mapeando os termos da consulta e, assim, servindo como uma ferramenta para a indexação, como um controle de vocabulário, por 
exemplo; e, por fim, desempenhar o papel de um dicionário mono/bi/multilíngue, inclusive no processamento de linguagem natural e tradução (SOERGEL, 1999).

Direcionando às operações da classificação aos ambientes digitais, como websites, bases de dados, bibliotecas digitais e repositórios institucionais, questões ocorrem sobre design de pesquisa/recuperação de informações, análise facetada, navegação hierárquica, indexação e linguagem natural, como um cenário discursivo e aplicável da arquitetura da informação ( $\mathrm{Al}$ ), ao elencar aspectos de representação, organização, navegação e busca. Entre os quatro sistemas, ou componentes, destaca-se a organização, pois é o aspecto que discute e realiza a classificação em websites, como o passo natural para estrutura-los ${ }^{1}$, ou seja, a forma como o design de suas interfaces será construído a partir da influência de um arranjo sistêmico que delineará a classificação das informações e suas relações conceituais, as tornando propícias à recuperação.

O desenvolvimento de uma interface será o reflexo do trabalho de design, encarado no presente artigo como a etapa em que se molda uma estratégia de alto nível na Al de um website, seja na criação de modelos, wireframes e/ou esquema de metadados. Esta fase é geralmente a que os arquitetos da informação possuem mais trabalho, mas a quantidade não pode ir contra a qualidade, pois a má execução do design pode arruinar a melhor estratégia (ROSENFELD; MORVILLE; ARANGO, 2015). Diante de tamanha importância, independentemente do tipo de trabalho, o design deve atrair, em grande parte, a intuição, tanto quanto possível, conforme as regras enquadradas pelo intelecto humano (RANGANATHAN, 1961).

Trazendo a fala do indiano Shiyali Ramamrita Ranganathan (1892-1972), de 1961 para os tempos atuais, tem-se o design com foco na informação, preocupado com a forma como os conteúdos serão organizados. Em websites de e-commerce, por exemplo, é comum identificar SOC hierárquicos, facetados e navegáveis, o que permite ir ao encontro da teoria da classificação facetada (TCF) de Ranganathan. ParaDahlberg (1979) e Satija (2017), entre as suas

\footnotetext{
${ }^{1}$ Neste artigo, estruturar significa "organizar as diferentes partes que constituem uma estrutura (um todo), conforme um plano ou sistema; construir [...]. [...] Planejar algo de forma cuidadosa e pormenorizada [...] (MICHAELIS, 2020, online).
} 
contribuições estão os três níveis (planos) de classificação, sendo o das ideias (conceitos, mensagens), o verbal (expressão verbal dos conceitos) e 0 notacional (conceitos em formas de símbolos, sinais, letras, números etc.).

Considerando o primeiro plano como um nível superior, pois as palavras (linguagem) e os números apenas existem porque há uma ideia por trás de ambos, como manifestações do plano (CAMPOS, 2001), o ideacional apresentou-se como o fio condutor para estudar o uso de facetas na Ol adotada na Al de ambientes digitais. Para Ranganathan (1967), o objeto faceta é um termo genérico usado para denotar algum item, como um assunto básico ${ }^{2}$ ou uma ideia isolada ${ }^{3}$, além de formar renques ${ }^{4}$, termos e números. Isto é, uma faceta "[...] é reagida como uma manifestação de uma das cinco Categorias Fundamentais [...]" (RANGANATHAN, 1963, p. 1*25, grifo do autor), representadas pela mnemônica personalidade, matéria, energia, espaço e tempo (PMEST) (ibid, 1960, 1963, 1967). De uma maneira geral, personalidade $[P]$ é a característica unívoca do assunto, como um tipo específico de biblioteca, matéria $[\mathrm{M}]$ refere-se aos aspectos físicos que compõem o assunto, como livros de capa dura, energia [E] é a ação que ocorre com relação ao assunto, como a catalogação realizada nos livros, espaço [S] é o aspecto geográfico de localização do assunto, como o local de publicação do livro, e tempo [T] é o período associado ao assunto, como o ano de publicação da obra.

Pressupondo que um design interativo de interfaces pode ser influenciado pela classificação, neste caso, a partir de arranjos de facetas que direcionem a estruturação dos ambientes e potencializem a postura intuitiva dos usuários, como um cenário de investigação e desenvolvimento assumido na Al, este artigo, a partir da técnica de pesquisa exploratória, quanto aos anseios da investigação,

\footnotetext{
2 É um elemento sistematizado de ideias correspondentes a um campo (RANGANATHAN, 1967), ou seja, são "[...] áreas mais abrangentes do conhecimento, como Matemática, Agricultura" (CAMPOS, 2001, p. 49).

${ }^{3}$ Elemento que forma um componente de um assunto, mas que não pode ser considerado um assunto em si. Campos (2001) cita a ideia isolada "Milho", que ao ser combinada com o assunto básico "Agricultura" define-se o assunto "Agricultura do Milho".

4 Os renques são arranjos em "[...] séries horizontais. Por exemplo: Macieira e Parreira são elementos da Classe Árvore Frutífera, formada pela característica de divisão - tipo de árvores frutíferas" (CAMPOS, 2001, p. 51).
} 
e pelo levantamento bibliográfico, o que permitiu identificar ambientes digitais que fazem uso de facetas, a partir da coleta de dados qualitativa, objetiva apresentar um panorama de uso de facetas na estruturação de ambientes digitais. Especificamente, objetiva-se elencar modelos de facetação, verificar suportes digitais onde os modelos de facetação foram adotados, ilustrar a evolução dos ambientes digitais ao longo dos anos e identificar facetas à luz da mnemônica PMEST.

\section{ARQUITETURA DA INFORMAÇÃO: ESTRUTURAS DE ORGANIZAÇÃO}

A criação de interfaces se transformou em uma questão problemática a partir da ascensão da Internet (1990). Um pouco depois, em meados dos anos 1990, essa preocupação foi se deflagrando a partir dos grandes investimentos pelas empresas no desenvolvimento de seus ambientes digitais na rede mundial de computadores, pois,a interface, comumente chamada de tela, dá "[...] forma à interação entre usuário e computador. A interface atua como uma espécie de tradutor, mediando entre as duas partes, tornando uma sensível para a outra" (JOHNSON, 2001, p. 19).

Preocupados com as interfaces, "para reduzir custos de produção e manutenção, ergonomistas passaram a criar novas metodologias, que identificassem problemas relativos ao contexto de uso dos sistemas" (NASCIMENTO; AMARAL, 2010, p. 14). Quando esta preocupação atingiu os websites, marcou-se a evolução nas metodologias formais e multidisciplinares aplicadas nesses espaços, sendo as primeiras tentativas de adotar um conceito de Al. Para Manzotti (2013), esta preocupação deve-se a criação de websites sem planejamento, permeados por problemas de usabilidade, dificuldades de encontrabilidade e custo oneroso na alteração das interfaces, culminando na expressão $\mathrm{Al}$, referindo-se a uma metáfora análoga a construção de prédios.

Basicamente, Al pode ser vista como a "arte e a ciência de dar forma a produtos de informação e experiências para apoiar usabilidade e encontrabilidade" (ROSENFELD; MORVILLE; ARANGO, 2015, p. 24). Em um 
sentindo mais amplo, o campo da Al apresenta-se como um "[...] projeto de estruturas (ambientes informacionais) que fornecem aos usuários recursos necessários para transformar suas necessidades em ações e para atingir seus objetivos com sucesso [...]" (AGNER, 2009, p. 90, destaque do autor). Para tanto, reitera-se que a Al acontece a partir de um conjunto de sistemas, também chamados de componentes, entre os quais enfatizam-se os sistemas de organização (organization systems), responsáveis em definir "[...] 0 agrupamento e a categorização de todo o conteúdo informacional" (REIS, 2007, p. 71).

$\mathrm{Na} \mathrm{Al}$, os sistemas de organização estão em linha ao ato de classificar, ação que significa impactar nos processos de recuperação, a partir das seguintes sentenças sobre a informação desejada: "Quem vai usá-la? Por quê? Eles vão buscá-la, navegar ou ambos? Quão bem eles conhecem o assunto? Lembre-se sempre, é para eles usarem" (DENTON, 2011). Essa consciência, sobre a classificação, influenciará nas "exibições de estilo de diretório e estruturas de navegação para recursos on-line [...]" (BROUGHTON et al., 2005, p. 134).

Entre os sistemas de organização estão as chamadas estruturas de organização, por modelos de hierarquia, de hipertexto e de estruturas de banco de dados (BD) relacionais (ROSENFELD; MORVILLE; ARANGO, 2015), os quais seriam proporcionais aos SOC discutidos no contexto da OC. Esse paralelismo, entre estruturas de organização e SOC, é discutido por Hodge(2000) quando destaca um interesse significativo no uso de SOC para organizar os resultados de pesquisas na Internet, culminando em serviços de $\mathrm{OC}$ que facilitariam o acesso às fontes encontradas. Rosenfeld, Morville e Arango (2015) ainda enfatizam que cada estrutura possui pontos positivos ou negativos e que, por vezes, sugere-se que sejam usadas em conjunto, de forma complementar.

A estrutura de organização hierárquica (figura 1) é um modelo taxonômico que, a partir de algum critério de ordenação e associação, vem sendo discutida e desenvolvida, tanto em ambientes tradicionais, como as bibliotecas físicas, quanto nos espaços eletrônicos, tais como os portais 
institucionais e as bibliotecas digitais. Conforme ilustrado na hierarquia de plantas (figura 1), constata-se "[...] uma forma de representação do conhecimento disposta metaforicamente como uma árvore. Assim, essa hierarquia possui uma estrutura que se inicia em uma raiz [...] e depois itens dispostos em ramos [...]" (SCHIESSL; SHINTAKU, 2012, p. 80), isto é, as classes desta árvore são subdivididas e posteriormente ordenadas, no sentido do mais geral e movendo-se ao mais específico (REITZ, 2014). No exemplo da figura 1 é possível perceber que "[...] cada termo aparece em um só lugar. Este foi o original plano para a taxonomia biológica" (ROSENFELD; MORVILLE; ARANGO, 2015, p. 301).

Figura 1 - Hierarquia única (simples)

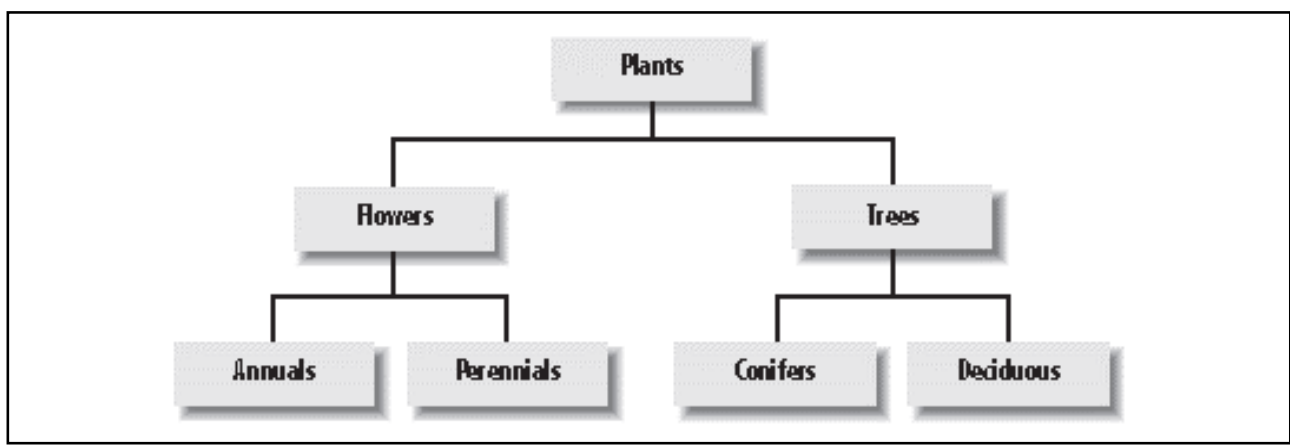

Fonte: Adaptado de Rosenfeld, Morville e Arango (2015, p. 117)

No hipertexto (figura 2) organiza-se a informação a partir de ligações entre textos, imagens etc., criando uma espécie de blocos de dados, e que estão interligados por alguma associação, como uma rede, inclusive permitindo o acesso e uso das informações de maneira não linear a partir de diferentes cenários de navegação (interpretações). Um ambiente que possui uma OI por hipertexto "[...] envolve dois principais tipos de componentes: os itens ou fragmentos de informações que serão vinculados e os links entre esses pedaços" (ROSENFELD; MORVILLE; ARANGO, 2015, p. 126). Os hipertextos, enquanto blocos de conteúdo, podem ser conectados, tanto hierarquicamente, quanto não hierarquicamente, ou ambos, desenhando uma rede de relacionamentos úteis e criativos. Porém, mesmo com essa flexibilidade, sem a apresentação de um contexto sobre os hipertextos, os usuários podem se sentir frustrados (ibid). 
Figura 2 - Rede de conexões hipertextuais

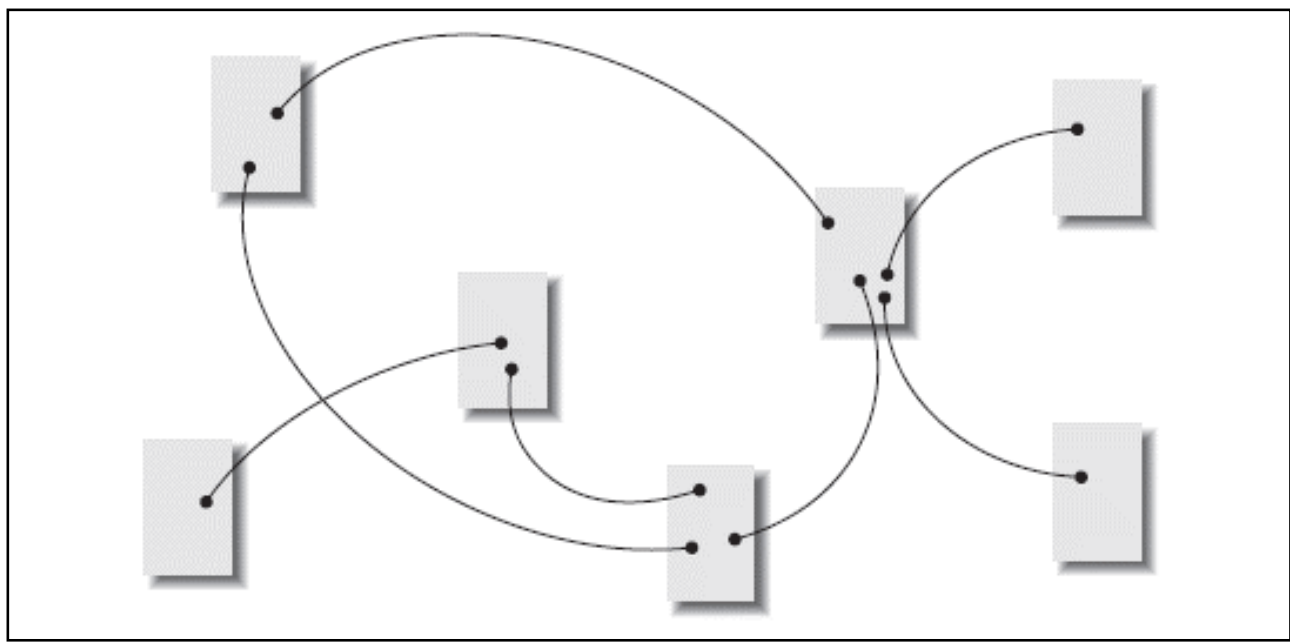

Fonte: Rosenfeld, Morville e Arango (2015, p. 126)

Quanto a organização por BD relacionais (figura 3), "[...] os dados são armazenados em um conjunto de relações ou tabelas. Linhas nas tabelas representam registros e colunas representam campos" (ROSENFELD; MORVILLE; ARANGO, 2015, p. 122). Esse modelo relacional se apresenta como "[...] uma coleção de dados dispostos para facilidade e velocidade de busca e recuperação [...]" (ibid) a partir das inúmeras possibilidades de consultas (relações) entre as tabelas, comumente usados em aplicações relativamente homogêneas, como catálogos de produtos e diretórios de pessoal (ibid).

Figura 3 -Elementos de um BD

\begin{tabular}{|c|c|c|c|c|}
\hline \multirow{2}{*}{$\begin{array}{l}\text { Emp } \\
\text { CódigoEmp }\end{array}$} & \multicolumn{2}{|c|}{ 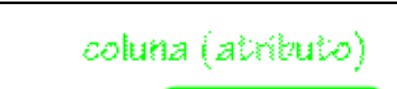 } & $\begin{array}{l}\text { nome do a almpo } \\
\text { nome do arrumulo }\end{array}$ & \\
\hline & Nome & CodigoDepto & CategFuncional & \\
\hline E5 & Souza & D1 & $\mathrm{C5}$ & \\
\hline E3 & Santos & D2 & $\mathrm{C5}$ & 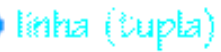 \\
\hline $\begin{array}{l}\mathrm{E} 2 \\
\mathrm{E} 1\end{array}$ & $\begin{array}{l}\text { Silva } \\
\text { Soares }\end{array}$ & $\begin{array}{l}\text { D1 } \\
\text { D1 }\end{array}$ & $\frac{C 2}{-}$ & \\
\hline & & $\begin{array}{l}\text { yalor de } \\
\text { valor de }\end{array}$ & 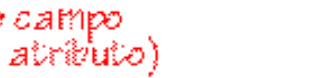 & \\
\hline
\end{tabular}

Fonte: Heuser (1998, p. 87).

Diante das estruturas de organização destacadas, enquanto espaços passíveis de crescimento, deve-se adotar uma hierarquia ampla e superficial ao invés de uma proposta estreita e profunda, o que permitiria a adição de conteúdos sem maiores reestruturações. Neste caso, interpreta-se que, ao se 
projetar uma estrutura de organização, não se deve estar limitado ao modelo hierárquico, sendo um começo para o arranjo das informações (ROSENFELD; MORVILLE; ARANGO, 2015) facetadas realizado na contemporaneidade, especialmente em websites de e-commerce como as livrarias digitais.

\subsection{ARQuITETURA da INFORMAÇão: EstrutURAS de OrGanizaÇÃo}

Uma Al efetiva deve mapear estruturas de sistemas humanos reais, exigindo-se que a prática de design seja hospitaleira para mudar conforme surjam necessidades de atualização. Neste caso, elenca-se o sistema de classificação facetado, como uma taxonomia essencialmente multidimensional, a qual pode representar, mais de perto, a estrutura do conhecimento real. Um esquema facetado baseia-se na abordagem contextual e não em perspectivas racionais ou empíricas, pois adota o que funciona melhor para cada situação (LOUIE; MADDOX; WASHINGTON, 2003).

Os arquitetos da informação precisam compreender que em um único SOC deve-se buscar o equilíbrio entre a exclusividade e a inclusividade. Neste cruzamento, as taxonomias facetadas ${ }^{5}$ são úteis quando se lida com grandes sistemas de informação, fazendo com que as taxonomias únicas deem lugar ${ }^{6}$ às abordagens mais flexíveis (ROSENFELD; MORVILLE; ARANGO, 2015).

A classificação facetada é um conjunto de classes chamado por Ranganathan de categorias fundamentais, mutuamente exclusivas e conjuntamente exaustivas. Essas categorias permitem que se isole uma perspectiva sobre o objeto, ou seja, define-se uma faceta. Em seguida, essas facetas poderão ser combinadas para representar os objetos. Desta forma, a taxonomia única adotada na época pelo tradicional diretório Yahoo! (Yahoo model) passou a ser fragmentada em taxonomias múltiplas (faceted model), concentradas em diferentes dimensões sobre o mesmo conteúdo (figura 4).

\footnotetext{
${ }^{5}$ Sinônimo de taxonomia multidimensional, múltipla, também chamada de poli hierárquica.

6 Morville e Rosenfeld (2006) defendiam que as facetas seriam o futuro dos arquitetos da informação, pensamento que foi mantivo em conjunto com Arango (2015).
} 
Figura 4 - Hierarquias única x múltipla (facetadas)

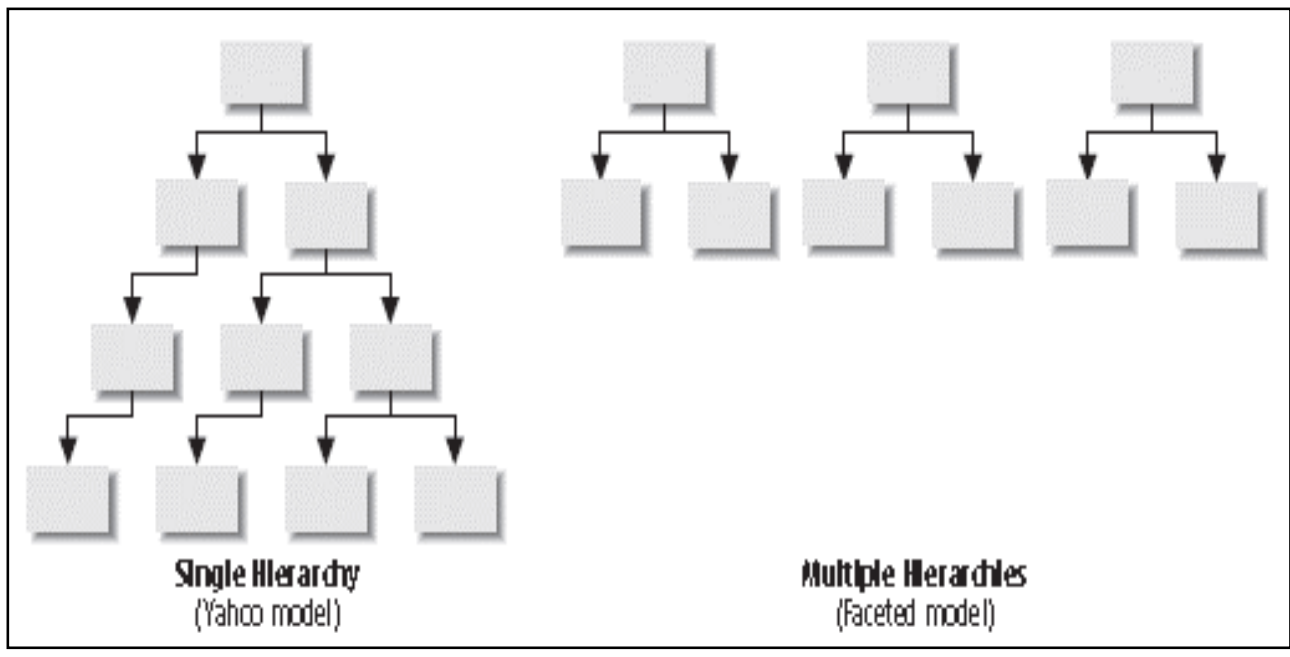

Fonte: Rosenfeld, Morville e Arango (2015, p. 304).

Ainda conforme a figura 4 , a taxonomia múltipla vai ao encontro da TCF de Ranganathan, este motivado em criar um sistema de classificação baseado "[...] na noção de que documentos e objetos tenham múltiplas dimensões, ou facetas" (ROSENFELD; MORVILLE; ARANGO, 2015, p. 303). Pelo fato de cada faceta estar focada em uma dimensão específica, a taxonomia pode ser menos profunda e mais plana, o que normalmente não ocorre nos tradicionais conceitos de taxonomia, exigindo um certo nível de especificidade/profundidade.

Considerar as facetas como uma maneira natural de organizar as coisas tornou-se uma redescoberta na estruturação dos ambientes digitais pelos desenvolvedores web e arquitetos da informação ao se perguntarem: "Que outras maneiras as pessoas querem ver esses dados? Qual seria a outra maneira de fatiá-los?". Porém, salienta-se que não passava de uma boa ideia pelos bibliotecários, sem a certeza de como usar as facetas nesses ambientes, enquanto os profissionais da web já o faziam, por vezes inconscientes ao Ranganathan e ao Classification Research Group (CRG) (DENTON, 2011).

\footnotetext{
${ }^{7} \mathrm{Na} \mathrm{Al}$, taxonomiaé separar informações em seções, partes, blocos ou menus, com um mínimo nível de especificidade,simplificando os conceitos normalmente adotados na OC. Taxonomia na Al é compreendida como um arranjo hierárquico de categorias quefaz parte da interface de um website, como uma árvore genealógica. Porém, quando esse conceito remete-se areunir informações em blocos e que estes sejam navegáveis, taxonomia passa a ser o ponto inicial do processo de design, de estruturar o website, aproximando-se de uma forma de Ol que seja inerente ao ser humano, o que cria o contexto últil aos usuários para que se sintam confortáveis (familiarizados) ao navegarem no ambiente (ROSENFELD; MORVILLE; ARANGO, 2015).
} 
La Barre (2006) já afirmava que as técnicas facetadas estavam sendo utilizadas na concepção de websites, o que motivou o desenvolvido da extensão XFML", como um Extensible Markup Language (XML) "[...] simples para trocar metadados sob a forma de hierarquias facetadas, às vezes chamadas de taxonomia. Os blocos [...] são tópicos, também chamados de categorias"(DIJCK, 2003), ilustrados na figura 5 , por exemplo.

Figura 5 - Scripts em XFML sobre detergentes para pratos



Fonte: Denton (2011).

Esta linguagem de marcação, de Peter Van Dijck's, considerada como uma das formas de implementar um sistema de classificação em computadores, constitui-se de duas tags básicas: facet e topic. A primeira tag citada representa a faceta maior, chamada de "brand_name" (marca) de detergentes para pratos, e a segunda define os focos da faceta, ou seja, as marcas "cascade", "electrasol", "ivory", "no_name", "palmolive" e "presidentes_choice" (DENTON, 2011), conforme ilustrado na figura 5 .

Outra opção de implementar um sistema de classificação em computadores seria via uma modelagem de $\mathrm{BD}$, especificamente pelo modelo de entidade-relacionamento (MER) do chinês Peter Chen. Nesta modelagem, cada faceta representa uma entidade (tabela) que, por sua vez, se relaciona com outras a partir da cardinalidade de um-para-muitos, em sua maioria. Por

8 Exchangeable Faceted Metadata Language. Wilson (2006) já defendia que Ranganathan é pode ser considerado o pai do tagueamento (marcação), pois a essência de sua contribuição é permitir a definição de qualquer notação de classificação para um dado recurso, o que soa mais como um ato de etiquetar do que uma classificação facetada propriamente dita. 
exemplo, cria-se uma entidade chamada "BRAND_NAME_T", fundamentada na faceta "marca" e que receberá os tipos de detergentes (DENTON, 2011), representada na figura 6 .

Figura 6 - Entidade "marca” no BD sobre detergentes para pratos

\begin{tabular}{|l|l|}
\hline PK & BRAND_NAME \\
\hline 1 & Cascade \\
\hline 2 & Electrasol \\
\hline 3 & Ivory \\
\hline 4 & No Name \\
\hline 5 & Palmolive \\
\hline 6 & President's Choice \\
\hline
\end{tabular}

Fonte: Denton (2011)

Diante das estruturas de organização apresentadas, as facetas se tornaram uma opção em ambientes digitais, especialmente em websites, tornando-se uma vertente discutida na Al e, assim, ocorrendo quase que de maneira indissociável ao planejamento de taxonomias que buscam pela multiplicidade de caminhos nos processos de navegação e busca. Neste sentido, estruturas de facetação foram desenvolvidas, como as elencadas no quadro 1. Contudo, em termos de $\mathrm{Al}$, os modelos de facetação estão alinhadas ao conjunto de categorias fundamentais do mundo dos negócios, idealizado por Rosenfeld, Morville e Arango (2015), especialmente nos websites de e-commerce.

\section{Quadro 1 - Estruturas de facetação ${ }^{9}$}

\begin{tabular}{|c|c|c|c|}
\hline Objeto/Foco & $\begin{array}{l}\text { Expressão de } \\
\text { navegação e } \\
\text { busca }\end{array}$ & Modelo & $\begin{array}{c}\text { Categoria } \\
\text { Fundamental }\end{array}$ \\
\hline \multirow{5}{*}{$\begin{array}{l}\text { Premiação } \\
\text { Nobel } \\
\text { (1901-2004) }\end{array}$} & \multirow{5}{*}{ Winners } & \multirow{5}{*}{$\begin{array}{l}\text { FLexible information } \\
\text { Access using MEtadata in } \\
\text { Novel COmbinations } \\
\text { (FLAMENCO) (2006) }\end{array}$} & Gender \\
\hline & & & Country \\
\hline & & & Affiliation \\
\hline & & & Prize \\
\hline & & & Year \\
\hline \multirow{3}{*}{ Vinho } & \multirow{3}{*}{$\begin{array}{l}\text { Californian merlot } \\
\text { e riesling }\end{array}$} & \multirow{3}{*}{$\begin{array}{l}\text { La Barre (2006); Rosenfeld, } \\
\text { Morville e Arango (2015) }\end{array}$} & Type \\
\hline & & & Region (origin) \\
\hline & & & Winery (manufacturer) \\
\hline
\end{tabular}

${ }^{9}$ Os dados foram coletados em 2017 e conferidos nos anos seguintes até 2020. 
Márcio Bezerra da Silva, Zeny Duarte de Miranda Investigação panorâmica sobre a adoção de estruturas facetadas no viés da arquitetura da informação

\begin{tabular}{|c|c|c|c|}
\hline & & & Year \\
\hline & & & Price \\
\hline \multirow{3}{*}{ Vinho } & \multirow{3}{*}{ Wine } & \multirow{3}{*}{ Sistema Facetmap (2009) } & Browse varietal \\
\hline & & & Browse region \\
\hline & & & Browse price \\
\hline \multirow{6}{*}{ Vinho } & \multirow{6}{*}{ Wine } & \multirow{6}{*}{ Denton (2011) } & Colour \\
\hline & & & Place \\
\hline & & & Kind of grape \\
\hline & & & Year of vintage \\
\hline & & & Quality \\
\hline & & & Price \\
\hline \multirow{6}{*}{$\begin{array}{l}\text { Mundo dos } \\
\text { negócios }\end{array}$} & \multirow{6}{*}{ Business world } & \multirow{6}{*}{$\begin{array}{l}\text { Rosenfeld, Morville e } \\
\text { Arango (2015) }\end{array}$} & Topic \\
\hline & & & Product \\
\hline & & & Document type \\
\hline & & & Audience \\
\hline & & & Geography \\
\hline & & & Price \\
\hline \multirow{7}{*}{ Vinho } & \multirow{7}{*}{$\begin{array}{l}\text { Cabernet } \\
\text { sauvignon }\end{array}$} & \multirow{7}{*}{ wine.com (2017-2020) } & Varietal \\
\hline & & & Region \\
\hline & & & Rating \& Price \\
\hline & & & Reviewed by \\
\hline & & & Size \& Type \\
\hline & & & Fine wine \\
\hline & & & Vintage \\
\hline \multirow{3}{*}{$\begin{array}{l}\text { Produtos de } \\
\text { informática }\end{array}$} & \multirow{3}{*}{ Desktop } & \multirow{3}{*}{$\begin{array}{l}\text { PC Connection (2017- } \\
2020)\end{array}$} & Category \\
\hline & & & Manufacturer \\
\hline & & & List price \\
\hline \multirow{6}{*}{ Culinária } & \multirow{6}{*}{ Chocolate } & \multirow{6}{*}{ Betty Crocker (2017-2020) } & Content type \\
\hline & & & Course \\
\hline & & & Meal type \\
\hline & & & Recipe rating \\
\hline & & & Total time \\
\hline & & & Prep time \\
\hline \multirow{4}{*}{$\begin{array}{l}\text { Produtos de } \\
\text { fotografia }\end{array}$} & \multirow{4}{*}{ Desktop } & & Price \\
\hline & & Camera House (2017- & Category \\
\hline & & 2020) & Brand \\
\hline & & & Cashback \\
\hline & & & What \\
\hline Repositório & Monument & Archaeology Data Service & Where \\
\hline digital & Miomumem & (ADS) $(2017-2020)$ & When \\
\hline & & & Resource \\
\hline & & & Refine your search \\
\hline & & & Content type \\
\hline & & Universidade Estadual da & Discipline \\
\hline Catálogo & $\begin{array}{l}\text { raceted } \\
\text { classification }\end{array}$ & Carolina do Norte (UECN) & Subject terms \\
\hline & & $(2017-2020)$ & Publication date \\
\hline & & & Language \\
\hline & & & Library location \\
\hline & & & Instituições \\
\hline & & & Repositório \\
\hline Biblioteca digital & & Instituto Brasilerro de & Programa \\
\hline $\begin{array}{l}\text { de teses e } \\
\text { dissertacões }\end{array}$ & $\begin{array}{l}\text { fassimicaçao } \\
\text { facetada }\end{array}$ & $\begin{array}{l}\text { Intormaçao em clencla e } \\
\text { Tecnologia (IBICT) }\end{array}$ & Autor \\
\hline (BDTD) & & (2017-2020) & Contribuidor \\
\hline & & & Orientador/a \\
\hline
\end{tabular}




\begin{tabular}{|c|c|c|c|}
\hline & & & Idioma \\
\hline & & & Assunto \\
\hline & & & Assunto em inglês \\
\hline & & & Área do Conhecimento \\
\hline & & & Ano de Defesa \\
\hline & & & Categoria \\
\hline & & & Autor/Colaborador \\
\hline & & & Encadernação \\
\hline Livraria diaital & Ciência da & Cultura(2017-2020) & Formato \\
\hline Livialla uigrial & Informação & Cuttura( $2017-2020)$ & Idioma \\
\hline & & & País de Produção \\
\hline & & & Ano de Edição \\
\hline & & & Fornecedor \\
\hline
\end{tabular}

Fonte: Elaborado pelos autores (2020)

As facetas e a web se combinam, pois, enquanto as primeiras criam um esquema organizacional multidimensional, os browsers são vistos como uma ferramenta de uso familiar à navegação em muitas dimensões (DENTON, 2011). Contextualizando, cita-se a dinâmica que vem sendo adotada em tempos recentes, ou seja, os itens de uma taxonomia são alterados a cada consulta realizada, demonstrando uma relação de ocorrência entre a busca e a posterior organização dos resultados, como acontece nas interfaces dos websites de ecommerce e na BDTD, por exemplo. Será justamente essa organização que, além de arranjar as informações na taxonomia, assumirá a função de navegação, características das chamadas taxonomias navegacionais ou facetadas.

\section{RESULTADOS}

Com a preocupação na estruturação de websites se tornando latente, no ano de 2005, o website de e-commerce de vinhos, chamado de wine.com, passou a ser encontrado na literatura sobre Al como um exemplo de aplicação na web. Entretanto, uma significativa parcela dessa valorização deve-se a sua forma inovadora, até então, de adotar taxonomias facetadas. Sendo mais explícito, assim como ilustrado na figura 7, Denton (2011) expôs que, diferentemente de elaborar uma lista contendo todos os vinhos, a qual seria longa, poder-se-ia olhar para os vinhos conforme as suas características. $\mathrm{Na}$ visão corroborativa de Rosenfeld, Morville e Arango (2015), existem diferentes olhares sobre o mesmo objeto, ou seja, sobre vinho. Em outras palavras, o vinho 
possui várias facetas que comumente são combinadas no momento da escolha.

Figura 7 - Facetação sobre vinho

\begin{tabular}{|c|c|}
\hline Facet & Sample controlled vocabulary values \\
\hline Type & $\begin{array}{l}\text { Red (Merlot, Pinot Noir), White (Chablis, } \\
\text { Chardonnay), Sparkling, Pink, Dessert }\end{array}$ \\
\hline Region (origin) & Australian, Californian, French, Italian \\
\hline $\begin{array}{l}\text { Winery } \\
\text { (manufacturer) }\end{array}$ & Blackstone, Clos du Bois, Cakebread \\
\hline Year & $1969,1990,1999,2000$ \\
\hline Price & $\begin{array}{l}\$ 3.99, \$ 20.99,<\$ 199, \text { Cheap, Moderate, } \\
\text { Expensive }\end{array}$ \\
\hline
\end{tabular}

Fonte: Rosenfeld, Morville e Arango (2015, p. 304-305)

Na web, não adotar a facetação seria o mesmo que percorrer tela após tela, dificultando a busca. Sendo assim, pode-se pensar no vinho em diferentes perspectivas, pois ele possui uma "cor", vem de um algum "lugar", é feito por um "tipo" (ou misturado) de uva, possui um "ano" de colheita (vintage), assume uma garantia de "qualidade" pelas autoridades vitivinícolas do seu país, vem em um "recipiente" (volume) e tem um "preço". Configura-se, então, um conjunto de categorias que combinarão para representar os vinhos ofertados (DENTON, 2011) e que estarão organizadas em taxonomias múltiplas, ao passo que estruturam a Al do website wine.com.

Figura 8 - Hierarquias múltiplas (facetadas) sobre vinho

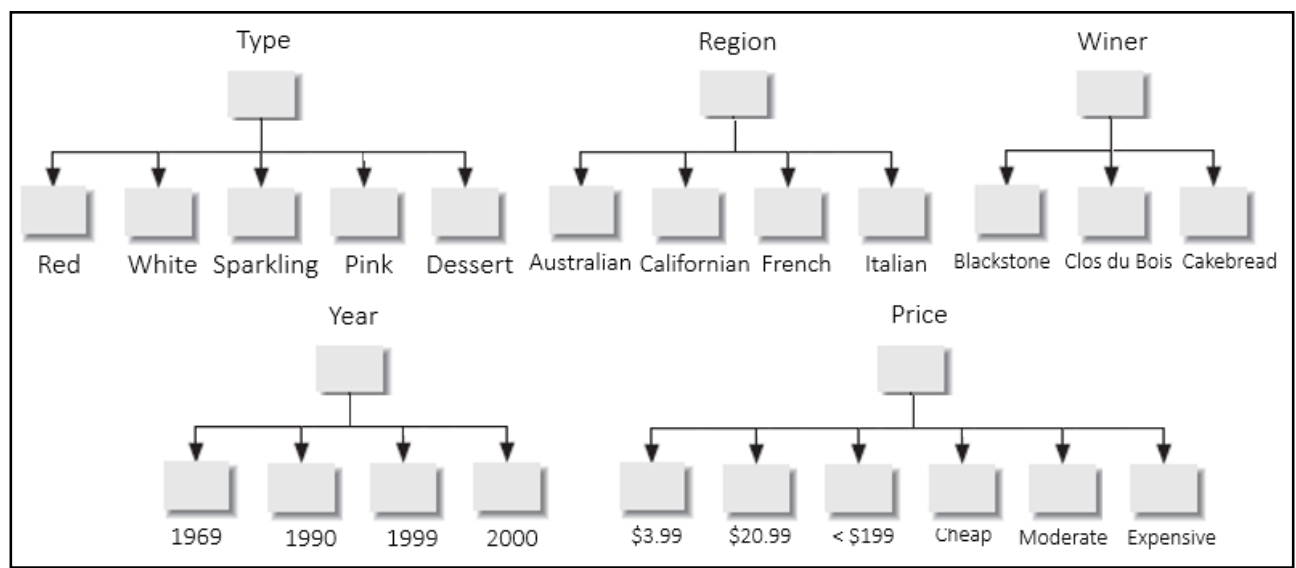

Fonte: Rosenfeld, Morville e Arango (2015, p. 304-305) e adaptado pelos autores (2017). 
Conforme a taxonomia facetada apresentada na figura 8, novas categorias fundamentais foram adotadas pelo website, assim como as elencadas no quadro 1 "varietal", "reviewed by", "size \& type" e "fine wine", enquanto outras foram renomeadas, como "year", passando para "vintage", e "price", se tornando "rating \& price". Especificamente, a figura 9 ilustrada essas categorias fundamentais e algumas de suas respectivas facetas, como, por exemplo, a categoria "varietal" e as correspondentes "red wine" e "rosé wine".

Figura 9 - Facetação no website wine.com

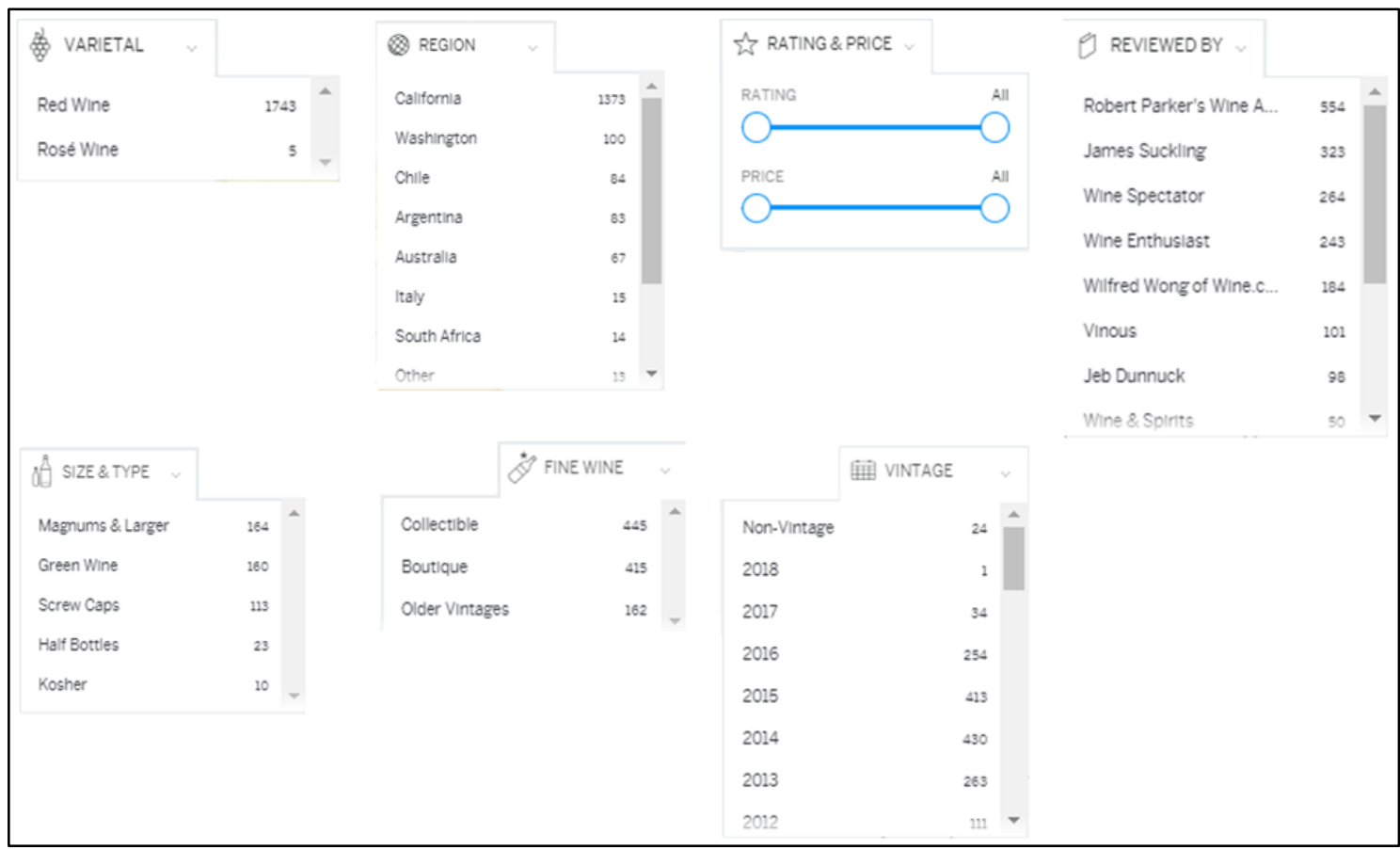

Fonte: wine.com (2017-2020)

Apesar da ideia preliminar de que as estruturas de facetação sejam, naturalmente, identificadas em websites, pela pesquisa estar concentrada no prisma da $\mathrm{Al}$, outros suportes de aplicação foram citados na literatura. Portanto, assim como ilustrado no gráfico 1, dos 12 formatos digitais identificados, 75\% (9) estão em linha ao mundo dos negócios, ou seja, websites de e-commerce. A porcentagem restante $(25 \%)$ assumiu uma plataforma cada, entre biblioteca digital, catálogo eletrônico e repositório digital, mostrando que, das 12 plataformas identificas, $83 \%$ corresponde ao mundo dos negócios, enquanto $17 \%$ é alusivo ao uso de facetas na Ol em ambientes acadêmicos. 


\section{Gráfico 1 - Suportes de aplicação na Al}

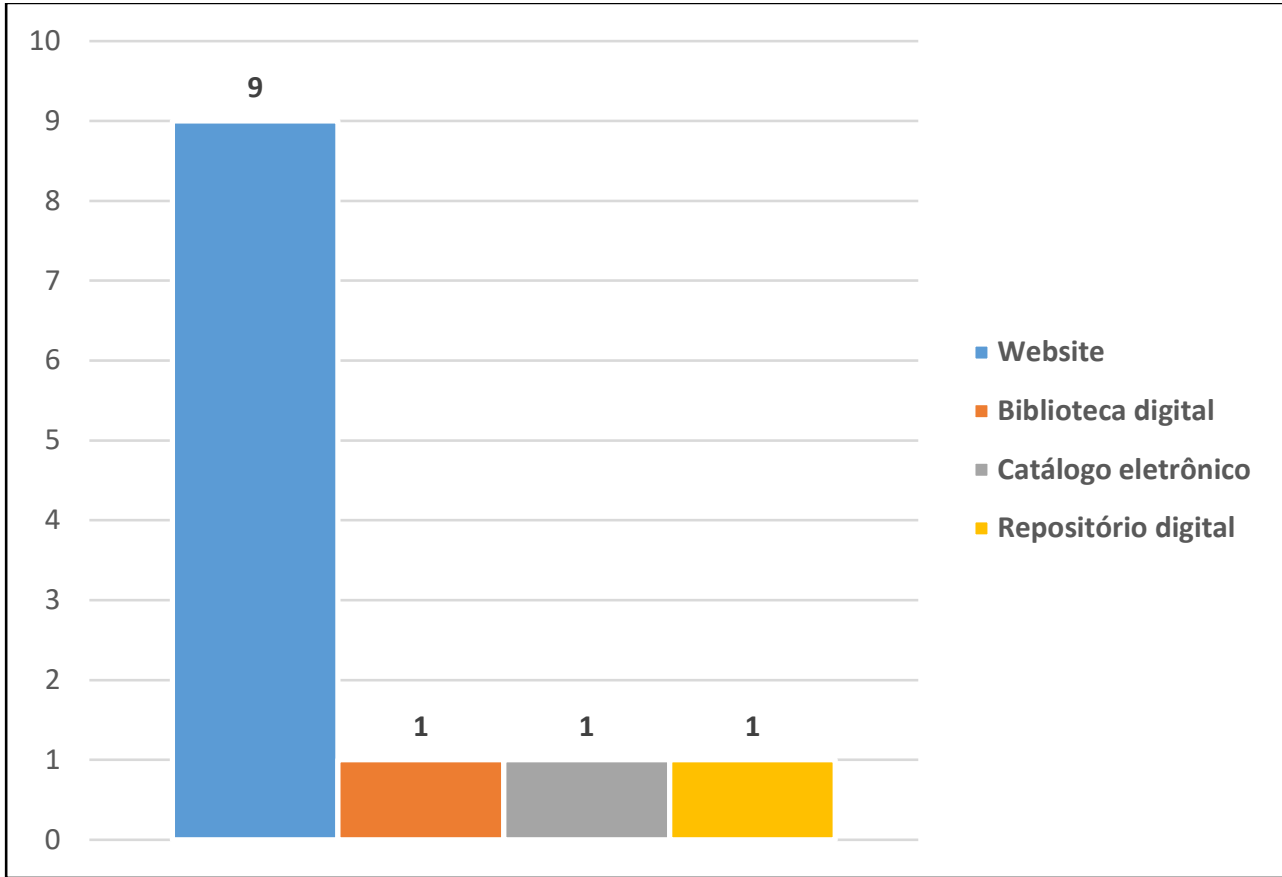

Fonte: Da pesquisa (2017-2020)

No que se refere ao seguimento evolutivo dos SOC facetados, no campo da Al foi possível perceber que a linha se inicia em 2006 e continua crescendo em quantidade com o passar dos anos, inclusive mostrando uma linha constante a partir do ano de 2017, conforme ilustrado no gráfico 2.

Gráfico 2 - Evolução dos ambientes na Al ao longo dos anos

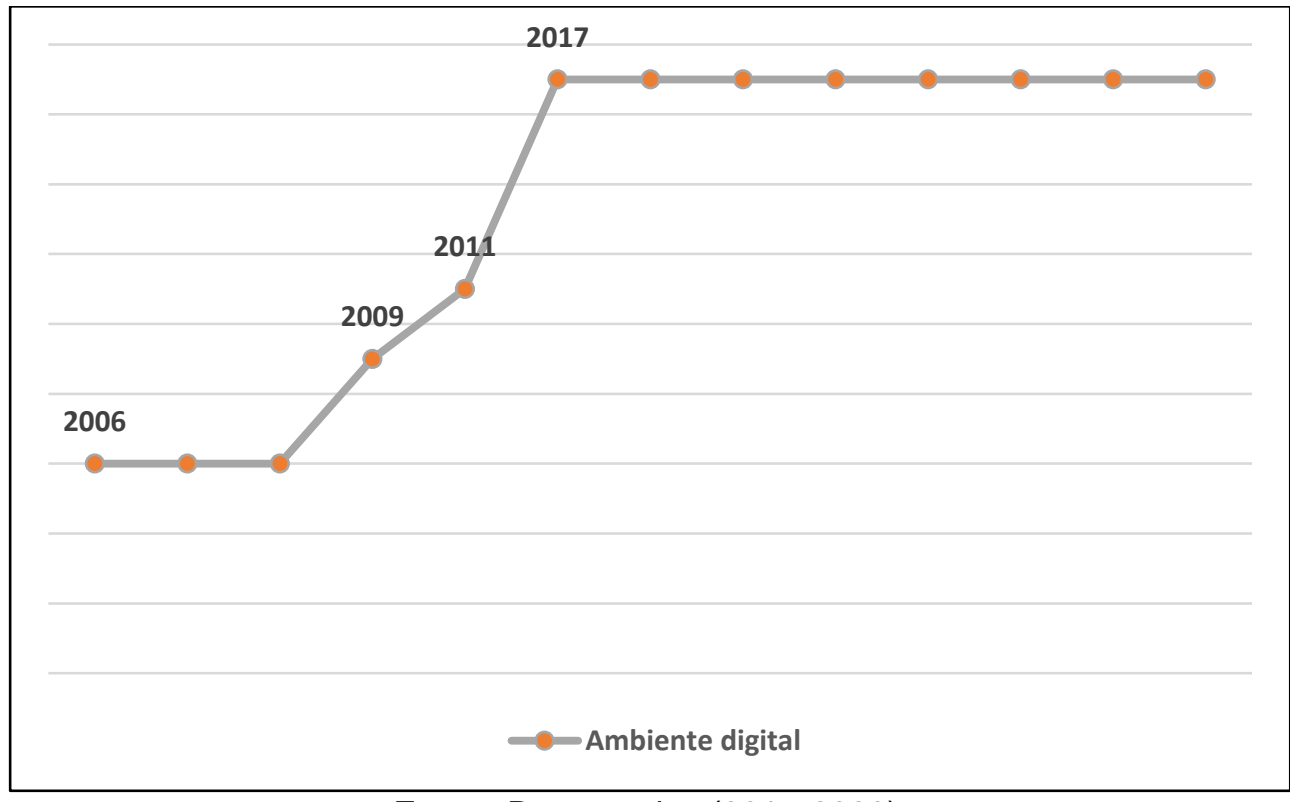

Fonte: Da pesquisa (2017-2020). 
Deve-se mencionar que alguns websites de e-commerce, como os de bebida, computadores e culinária, foram citados na literatura em 2006 e continuam sendo disponibilizados na Internet, alterando o design das interfaces conforme as tendências estruturais de cada época. Também deve-se mencionar que o website de e-commercePC Connection ${ }^{10}$ apresentou alterações no esquema de classificação de seus produtos, incluindo novas categorias fundamentais, alteradas conforme a busca do usuário e identificadas a partir de 2018, ao invés da sequência útil (padrão) observada e estudada em 2017.

Em sentido complementar ao gráfico 3 , no total de categorias fundamentais identificadas no prisma da Al (quadro 1), ao serem comparadas a mnemônica PMEST (gráfico 4), a categoria [P] possui a maior quantidade de citações, correspondendo a 55\% (41), enquanto que a parcela minoritária se refere a categoria [E], quantificada em $1 \%(1)$ do total.

\section{Gráfico 3 - Categorias fundamentais na Al à luz do PMEST ${ }^{11}$}



Fonte: Da pesquisa (2017-2020).

10 De 2017 até 2020, novas representações foram surgindo, na busca em especificar o produto pesquisado. A partir da busca surge a sequência útil (padrão) do website, como chamava Ranganathan, ou seja, as categorias que são exibidas para todos os produtos e que foram computadas no artigo. A partir de 2018 foram surgindo categorias específicas, como " $56 \mathrm{~K}$ technology", "adapter type", "attributes", "audio input types", "audio output mode" etc., e representações ainda mais especificas por produto, identificadas pelo uso do símbolo cerquilha (\#). Na busca pelo produto "desktop", as representações "especiais" são "\#of bays (open)", "\#of bays (total)", "\#ports", "\#processors installed", "\#processors supported", "\#RAM modules".

11 Valores: $55 \%$ do total corresponde a [P], 17\% a [M], 1\% a [E], 14\% a [S] e $13 \%$ a [T]. 
No caso da categoria $[E]$, trata-se de uma perspectiva não comum no viés dos usuários em ambientes de e-commerce, pois o foco está no produto e não na ação, como as atividades que levaram a fabricação de certo objeto, por exemplo. Por outro lado, a categoria [E] pode apresentar um resultado diferente em ambientes de práticas acadêmico-profissionais, como nas bibliotecas, pois as obras têm suas disponibilizações justificadas pelas ações preliminares de catalogação, classificação e indexação.

No caso da categoria $[E]$, trata-se de uma perspectiva não comum no viés dos usuários em ambientes de e-commerce, pois o foco está no produto e não na ação, como as atividades que levaram a fabricação de certo objeto, por exemplo. Por outro lado, a categoria [E] pode apresentar um resultado diferente em ambientes de práticas acadêmico-profissionais, como nas bibliotecas, pois as obras têm suas disponibilizações justificadas pelas ações preliminares de catalogação, classificação e indexação.

\section{CONSIDERAÇÕES FINAIS}

O artigo apresentou um olhar sobre a estruturação no design de interfaces de websites a partir do uso de facetas, vislumbrando a influência da Ol em ações de navegação e busca no escopo do e-commerce, o que permite a inferência de que os problemas nos processos de recuperação não estão concentrados nas ferramentas de busca. $\mathrm{Na}$ verdade, acredita-se que esses problemas vão ao encontro da forma como ocorre a OI, ou seja, de como os conteúdos são classificadas a partir da observação de suas características, as quais aproximam e relacionam as informações.

A discussão travou-se na atividade da classificação por facetas, encarada como uma temática que encontra fomentos literários no âmbito da $\mathrm{OC}$, e que se propagou em outras áreas como é o caso da $\mathrm{Al}$, ao considerar a adoção de facetas na apresentação e na organização de conteúdos, que por sua vez impactarão no design da interface do ambiente digital.

Respondendo aos objetivos de elencar estruturas de facetação, exemplificar uma estrutura de facetação de um website de e-commerce, verificar 
suportes onde os ambientes de facetação foram adotados, ilustrar a evolução dos ambientes digitais ao longo dos anos e identificar facetas à luz da mnemônica PMEST, os resultados coletados mostraram que: os websites de $e$ commerce são os espaços onde os modelos facetados são mais adotados, tendo o wine.com como um modelo, inclusive sendo atualizado conforme as diferentes perspectivas que o objeto vinho recebe com o tempo; o escopo de criação e aplicação de facetações concentra-se no mundo dos negócios, iniciando em 2006 e continuando ao longo do tempo; e a categoria [P] foi a mais presente entre os itens da mnemônica PMEST, como parece ser um fato consolidado em taxonomias facetadas.

Concluiu-se que a adoção de facetas permite que um mesmo objeto tenha interpretações diferentes em um menu navegacional, formaliza a multidimensionalidade idealizada por Ranganathan a partir de mnemônicas personalizadas ao escopo do ambiente digital, orienta na construção de taxonomias múltiplas e rasas e representa uma forma de classificação que acentua-se ao longo dos anos, fortalecendo a inferência de que os websites assumiram a facetação como um caminho para organizar a informação segundo estruturas de navegação e busca integradas.

A referida conclusão reafirma as possíveis contribuições discursivas sobre o desenvolvimento e aplicação da TCF em ambientes digitais, proporcionando um cenário de incentivo a novos estudos quanto a adoção de facetas, nos mais variados tipos de SOC, assim como na estruturação do design de interfaces.

\section{REFERÊNCIAS}

AGNER, L. Ergodesign e arquitetura de informação: trabalhando com o usuário. 2. ed. Rio de Janeiro: Quartet, 2009.

ARCHAEOLOGY DATA SERVICE. ADS. 2020. Disponível em: http://archaeologydataservice.ac.uk/. Acesso em: 02 maio 2020.

BETTY CROCKER. General Mills. 2020. Disponível em: https://www.bettycrocker.com/. Acesso em: 02 maio 2020. 
BROUGHTON, V. et al. Knowledge Organization. European Curriculum Reflections on Library and Information Science Education, p. 133-148, 2005. Disponível:

http://arizona.openrepository.com/arizona/bitstream/10150/105851/1/Knowledg eOrg_chapter+7.pdf. Acesso: 12 abril 2020.

CAMERA HOUSE. Camera house. 2020. Disponível em: https://www.camerahouse.com.au/. Acesso em: 02 maio 2020.

CAMPOS, M. L. A. Linguagem documentária: teorias que fundamentam sua elaboração. Niterói: Ed. da UFF, 2001.

DAHLBERG, I. Knowledge organization: a new Science? Knowledge organization, v. 33, n. 1, p. 11-19, 2006.

DAHLBERG, I. Knowledge organization: its scope and possibilities. Knowledge Organization, v. 20, n. 4, p. 211-222, 1993.

DAHLBERG, I. Teoria da classificação, ontem e hoje. In: CONFERÊNCIA BRASILEIRA DE CLASSIFICAÇÃO BIBLIOGRÁFICA, Rio de Janeiro, 1972. Anais... Brasília, IBICT/ABDF, 1979. v. 1, p. 352-370. Disponível em: http://www.conexaorio.com/biti/dahlbergteoria/dahlberg_teoria.htm. Acesso em: 05 abr. 2020.

DAHLBERG, I. Teoria do conceito. Tradução de Astério Tavares Campos. Ciência da Informação, Brasília, v. 7, n. 2, p. 101-107, 1978. Disponível em: http://revista.ibict.br/ciinf/article/view/115/115. Acesso em: 05 abr. 2020.

DENTON, W. How to make a faceted classification and put it on the web. 2011. Disponível em: https://www.miskatonic.org/library/facet-web-howto.html. Acesso em: 02 maio 2020.

DIJCK, P. V. Introduction to XFML. O'Reilly Media, Inc., 2003. Disponível em: https://www.xml.com/pub/a/2003/01/22/xfml.htm. Acesso em: 09abr. 2020.

FACETMAP. Facetmap. 2009. Disponível em: http://www.facetmap.com/. Acesso em: 02 maio 2020.

FLEXIBLE INFORMATION ACCESS USING METADATA IN NOVEL COMBINATIONS. The Flamenco Search Interface Project. 2006. Disponível em: http://flamenco.berkeley.edu/. Acesso em: 02 maio 2020.

HEUSER, C. A. Projeto de Banco de Dados. 5. ed. UFRGS: Editora Sagra Luzzanato, 2004. (Série Livros Didáticos).

HJØRLAND, B. What is Knowledge Organization (KO)? Knowledge Organization, Copenhagen, v. 35, n. 2/3, 2008. Disponível em: https://www.ergon-verlag.de/downloads/35_2008_2-3.pdf. Acesso em: 20 maio 2020. 
HODGE, G. Systems of Knowledge Organization for Digital Libraries:

beyond traditional authorities files. Washington, DC: The Council on Library and Information Resources, 2000. Disponível em:

http://www.clir.org/pubs/reports/pub91/pub91.pdf. Acesso em: 15 maio 2020.

INSTITUTO BRASILEIRO DE INFORMAÇÃO EM CIÊNCIA E TECNOLOGIA. Biblioteca Digital de Teses e Dissertações. [2020?]. Disponível em:

http://bdtd.ibict.br/vufind/. Acesso em: 02 maio 2020.

JOHNSON, S. Cultura da interface: como o computador transforma nossa maneira de criar e comunicar. Rio de Janeiro: J. Zahar, 2001.

KELSCH, S. Ranganathan's Colon Classification in 1500 Words or Less. 2003. Disponível em: http://kelschindexing.com/colonclassification.html. Acesso em: 20 maio. 2020.

LIVRARIA CULTURA. Livraria cultura. 2020. Disponível em: https://www.livrariacultura.com.br/. Acesso em: 02 maio 2020.

LA BARRE, K. The use of faceted analytico-synthetic theory as revealed in the practice of website construction and design. 310f. Tese (Doutorado de Filosofia em Ciência da Informação) - Programa de Pós-Graduação em Ciência da Informação - Escola de Biblioteconomia e Ciência da Informação, Universidade de Indiana, Indiana, 2006.

LIMA, J. L. O.; ALVARES, L. Organização e representação da informação e do conhecimento. In: ALVARES, L. (Org.). Organização da informação e do conhecimento: conceitos, subsídios interdisciplinares e aplicações. São Paulo: B4 Editores, 2012.

LOUIE, A. J.; MADDOX, E. L.; WASHINGTON, W. Using Faceted Classification To Provide Structure For Information Architecture. IA Summit, 2003. Disponível em:

http://depts.washington.edu/pettt/presentations/conf_2003//ASummit.pdf. Acesso em: 15 maio 2020.

MICHAELIS. Dicionário Brasileiro da Língua Portuguesa. Melhoramentos, 2020. Disponível em: http://michaelis.uol.com.br/moderno-portugues/. Acesso em: 11abr. 2020.

MANZOTTI, C. S. Design de Interface em Dispositivos Móveis. Mackenzie, 2013. Disponível em:

https://issuu.com/cmanzotti/docs/design_de_interface_em_dispositivos. Acesso em: 10 nov. 2020.

NASCIMENTO, J. A. M.; AMARAL, S. A. Avaliação de usabilidade na Internet. Brasília: Thesaurus, 2010. 
PC CONNECTION. Connection. 2020. Disponível em:

https://www.connection.com. Acesso em: 02 maio 2020.

RANGANATHAN, S. R. Colon Classification. Bombay: Asia Publishing House, 1963.

RANGANATHAN, S. R. Colon Classification: basic classification. Bombay: Madras Library Association Publication, 1960.

RANGANATHAN, S. R. Prolegomena to library classification. Bombay: Asia PublishingHouse, 1967.

REIS, G. A. Centrando a arquitetura de informação no usuário. Dissertação (Mestrado em Ciência da Informação) - Escola de Comunicações e Artes Universidade de São Paulo, São Paulo, 2007. Disponível em: http://www.teses.usp.br/teses/disponiveis/27/27151/tde-23042007-141926/ptbr.php. Acesso em: 05 abr. 2020.

REITZ, J. M. Online Dictionary for Library and Information Science. Santa Barbara: ABC-CLIO, 2014. Disponível em: https://www.abcclio.com/ODLIS/odlis_A.aspx. Acesso em: 20 jun. 2020.

ROSENFELD, L. MORVILLE, P.Information architecture for the word wide web. 3. ed. Sebastopol: O'Reilly, 2006.

ROSENFELD, L. MORVILLE, P; ARANGO, J. Information architecture for the web and beyond. 5. ed. Sebastopol: O'Reilly, 2015.

SATIJA, M. P. Colon Classification (CC). Encyclopedia of Knowledge Organization. 2017. Disponível em: http://www.isko.org/cyclo/colon_classification. Acesso em: 20 maio 2020.

SCHIESSL, M.; SHINTAKU, M. Sistemas de organização do conhecimento. In: ALVARES, L. (Org.). Organização da informação e do conhecimento: conceitos, subsídios interdisciplinares e aplicações. São Paulo: B4 Editores, 2012.

SOERGEL, D. The rise of ontologies or the reinvention of classification. Journal of the American Society of Information Science, v. 50, n. 12, 1999, p. 1119-1120. Disponível em: http://www.dsoergel.com/cv/B70.pdf. Acesso em: 05 abr. 2020.

UNIVERSIDADE ESTADUAL DA CAROLINA DO NORTE. Summon: NSCU libraries. 2020. Disponível em: http://ncsu.summon.serialssolutions.com/\#!/. Acesso em: 02 maio 2020.

WILSON, T. The strict faceted classification model. Travis Wilson \& Facetmap, 2006. Disponível em: 
http://www.facetmap.com/pub/strict_faceted_classification.pdf. Acesso em: 02 maio. 2020.

WINE.COM. Wine.com. 2020. Disponível em: https://www.wine.com/. Acesso em: 02 maio 2020.

\title{
PANORAMIC INVESTIGATION ON THE ADOPTION OF FACETED STRUCTURES WITHIN THE VIEW OF INFORMATION ARCHITECTURE
}

\begin{abstract}
Introduction: Considering the information architecture organization systems, with emphasis onorganizational structures, the adoption of facets in digital environments is discussed, considering facet models, digital supports where the models were adopted, models by scope of action, evolution of digital environments over the years and facets in the light of the fundamental Personality-Matter-Energy-Space-Time categories, stemming from the faceted classification theory of the Indian Ranganathan. Objective:To present an overview of the use of facets in structuring digital environments.Methodology:exploratory and bibliographic study, with qualitative data collection from digitalenvironments that use facet models and their characteristics, occurred in 2017 and continued until 2020.Results:E-commerce websites are the spaces where faceted models are most adopted, with wine.com as an inspiration, including some updating their facets in new perspectives over time; the scope of application of factions is concentrated in the business world, starting in 2006 and continuing over time; and personality was the most present among the items of the fundamental categories.Conclusions:The adoption of facets allows the same object to have different interpretations in a navigational menu, formalizes the multidimensionality idealized by Ranganathan from mnemonics customized to the scope of the digital environment, guides in the construction of multiple and shallow taxonomies and represents a form of classification which has been accentuated over the years, strengthening the inference that websites have taken facet as a way to organize information according to integrated navigation and search structures.
\end{abstract}

Descriptors:Facets. Fundamental Categories. Faceted Classification Theory. Information Architecture. Organization Structures.

\section{INVESTIGACIÓN PANORÁMICA SOBRE LA ADOPCIÓN DE ESTRUCTURAS FACETADAS DENTRO DE LA VISIÓN DE LA ARQUITECTURA DE INFORMACIÓN}

\begin{abstract}
RESUMEN
Introducción:Considerando los sistemas de organización de la arquitectura de la información, con énfasis en las estructuras organizacionales, se discute la adopción de facetas en entornos digitales, considerando modelos de facetas, soportes digitales donde los modelos fueron adoptados, modelos por alcance de acción, evolución de
\end{abstract}


entornos digitales a lo largo de los años y facetas a la luz de las categorías fundamentales de Personalidad-Materia-Energía-Espacio-Tiempo, derivadas de la teoría de clasificación facetada del indio Ranganathan.Objetivo: Presentar una visión general del uso de facetas en la estructuración de entornos digitales. Metodología: Studio exploratorio y bibliográfico, con recopilación de datos cualitativos de entornos digitales que utilizan modelos facetarios y sus características, realizado en 2017 y continuado hasta 2020.Resultados: Los sitios web de comercio electrónico son los espacios donde los modelos facetados son los más adoptados, con wine.com como inspiración, in incluyendo algunas actualizaciones de sus facetas en nuevas perspectivas a lo largo del tiempo; El ámbito de aplicación de las facciones se concentra en el mundo de los negocios, comenzando en 2006 y continuando con el tiempo; y la personalidad fue la más presente entre los ítems de las categorías fundamentales.Conclusiones: La adopción de facetas permite que el mismo objeto tenga diferentes interpretaciones en un menú de navegación, formaliza la multidimensionalidad idealizada por Ranganathan a partir de mnemónicos personalizados para el alcance del entorno digital, guía en la construcción de taxonomías múltiples y superficiales y representa una forma de clasificación que se ha acentuado a lo largo de los años, fortaleciendo la inferencia de que los sitios web han tomado facetas como una forma de organizar la información de acuerdo con las estructuras integradas de navegación y búsqueda.

Descriptores: Facetas. Categorías fundamentales Teoría de clasificación facetada. Arquitectura informacional. Estructuras de organización.

Recebido em: 05.06.2020

Aceito em: 13.12 .2020 